\title{
Les dynamiques institutionnelles et les choix des responsables de projets du Campus Virtuel Suisse: une conciliation difficile
}

\section{Benedetto Lepori et Jean-François Perret}

Dans cet article, nous examinons le développement des projets du Campus Virtuel Suisse et leur interaction avec le contexte institutionnel. Nous montrons que le CVS et les hautes écoles ont des conceptions différentes concernant le concept et les modalités d'introduction de l'eLearning. Dans ce contexte, les projets sont amenés à se détacher partiellement du modèle proposé au début par le CVS et à développer des solutions originales, adaptées à chaque contexte d'application. A leur tour, ces expériences ont engendré au niveau du programme une révision des conceptions de l'eLearning, vers un modèle de cours hybride, et, dans une moindre mesure, de l'organisation du programme, avec l'introduction des centres de support universitaires. Maintes questions restent toutefois ouvertes, notamment en ce qui concerne le modèle organisationnel et économique.

\section{Introduction}

Au cours des années 1999 et 2000, le Campus Virtuel Suisse (CVS; www.virtualcampus.ch) a soutenu 50 projets de création de cours universitaires en ligne, réalisés par des consortia entre plusieurs hautes écoles suisses. Ces projets se trouvent actuellement à la fin de la phase de réalisation et, selon les plans prévus, les cours devraient commencer à être utilisés par les hautes écoles dans leurs programmes d'études.

Différentes études montrent que la plupart des projets se sont écartés sensiblement du modèle initial proposé par le CVS lors de la mise au concours du programme (Gurtner et al., 2004; Lepori \& Rezzonico, 2003). En effet, le CVS préconisait la réalisation de cours complètement en ligne, qui auraient été utilisés à distance par les étudiants de différentes universités. En réalité, seule un petit nombre de projets ont réalisé des cours accessibles sur Internet pour être suivis indépendamment des heures de cours. Dans la plupart des cas, cette mise à distance n'a été que partielle et les matériaux sont conçus plutôt comme des supports pour un cours en présence (éventuellement avec une réduction des heures 
d'enseignement). Par ailleurs, l'objectif initial d'un échange de modules d'enseignement entre les universités n'a été réalisé que partiellement: dans plusieurs cas, les projets sont composés de modules largement indépendants. Chacun d'eux est surtout utilisé par la haute école qui les a produits (Lepori \& Rezzonico, 2003). Finalement, il apparaît que la plupart des projets ont massivement sous-estimé le temps et les ressources nécessaires à la réalisation du cours (Gurtner et al., 2004) et que dans de nombreux cas, seule une partie des modules a été réalisée, ou certains modules ne sont pas encore traduits dans les différentes langues prévues pour le cours.

L'objectif de cet article est justement d'analyser, pour quelques aspects exemplaires, les modalités de réalisation des cours du Campus Virtuel Suisse et de les comparer avec les objectifs initiaux du programme. Ensuite, nous tenterons d'expliquer les décalages constatés par l'interaction entre les projets - conçus comme des acteurs avec leurs choix et leurs objectifs - et leur environnement, constitué notamment du CVS en tant que programme national, mais aussi des hautes écoles et, plus généralement, des acteurs de la politique universitaire en Suisse. Finalement, nous allons examiner dans quelle mesure le fonctionnement des projets a entraîné une révision des conceptions et des modèles de mise en œuvre de l'eLearning 1 au niveau du programme et des hautes écoles en général.

L'article est organisé en quatre parties. Dans la première section, nous indiquons les sources qui seront utilisées pour l'analyse. Ensuite, nous présentons un cadre conceptuel et nous analysons plus précisément les relations entre les projets et leur environnement. Dans la troisième section, nous focaliserons notre attention sur quelques thèmes choisis en considérant successivement les aspects suivants: le point de vue des acteurs; les choix qui ont été opérés au sein des projets; les apprentissages qui ont pu se produire au niveau institutionnel. Nous terminons par quelques remarques plus générales.

\section{Les sources}

Les résultats présentés dans cet article se basent sur des recherches réalisées depuis le début du CVS en 1999 dans le cadre de différents projets. Leur intérêt est de permettre une observation à différents niveaux du développement de l'eLearning en Suisse: le niveau de la politique universitaire et des organes directeurs des hautes écoles d'une part, celui du fonctionnement du programme et de ses relations avec les projets d'autre part et, finalement, celui des décisions et du développement des projets individuels.

Au cours des années 2002 et 2003, l'un d'entre nous a coordonné le mandat «Educational Management in the Swiss Virtual Campus» (EDUM; www.edum.ch), par lequel le CVS a demandé à l'Université de la Suisse italienne d'étudier les conditions organisationnelles et institutionnelles de l'introduction de l'eLearning dans les hautes écoles suisses. 
Dans le cadre de EDUM, nous avons examiné au cours de l'année 2002 dixhuit projets du CVS. Les projets ont été choisis, d'entente avec la direction du programme, parmi ceux qui étaient les plus avancés, et sont répartis entre tous les domaines scientifiques. Après une analyse documentaire, une interview a été menée avec le chef de projet et/ou le coordinateur sur la base d'un questionnaire qui leur était envoyé à l'avance. Le questionnaire touchait les points suivants:

- les objectifs du projet et la motivation pour introduire le eLearning;

- l'organisation du cours CVS, notamment le public cible visé et la relation entre enseignement en présence et enseignement à distance;

- les moyens nécessaires pour la production et le fonctionnement: le développement des contenus, la composition de l'équipe de projet, les ressources et les infrastructures nécessaires;

- les ressources financières et les perspectives de financement après la fin du soutien du CVS;

- les relations avec l'université et la faculté d'appartenance et avec le CVS.

Toutes les interviews ont été enregistrées et transcrites. Une analyse détaillée des résultats, comprenant aussi plusieurs transcriptions, a été publiée au cours de 2003 (Lepori \& Rezzonico, 2003); nous renvoyons le lecteur à ce rapport pour davantage de détails.

Ensuite, au cours de 2003, nous avons examiné l'état de développement du eLearning et les stratégies dans ce domaine dans les hautes écoles universitaires suisses (universités cantonales et EPF). Cette analyse a nécessité l'examen des documents fournis par les universités, la consultation des sites Web et des entretiens avec les responsables de l'eLearning des hautes écoles (au niveau rectorat et/ou centre de support). Les entretiens (sur la base d'un guide d'interview envoyé à l'avance) concernaient les sujets suivants: les objectifs et les stratégies des hautes écoles pour le eLearning, les activités existantes dans l'école et les mesures de soutien pour ces activités (financements disponibles; création d'un centre de support). Pour chaque entretien, un résumé a été préparé et envoyé aux intéressés pour vérification. Les résultats principaux, ainsi qu'une comparaison avec les pays voisins, sont publiés dans un rapport (Lepori \& Succi, 2003), ainsi que dans des présentations à des conférences (Lepori, Cantoni \& Succi, 2003). Cette étude a été récemment complétée par une analyse de la situation dans les hautes écoles spécialisées (Lepori \& Succi, 2004).

Cet article s'appuie d'autre part sur les travaux d'évaluation de deux projets du CVS. Au fur et à mesure de leur élaboration, les modules d'enseignement ont donné lieu à une série d'expérimentation (tests de terrain), dans différents contextes universitaires (Perret, 2004; Perret \& Schubauer-Leoni, 2004; Schubauer-Leoni \& Perret, 2003). Cette évaluation s'est centrée sur cinq dimensions relatives aux:

- Matériel de cours: Comment les étudiants apprécient-ils la clarté, l'apport, l'intérêt du matériel de cours? Le niveau de langage, les progressions adoptées et les éléments d'approfondissement sont-ils adaptés au public visé? 
- Dispositifs d'enseignement: La planification du cours; le rapport entre les temps de formation présentiels et les temps à distance; l'encadrement des étudiants; les possibilités de communication pédagogiques médiatisée (e-mail, forum) donnent-ils satisfaction?

- Facteurs contextuels: Les étudiants peuvent-ils consacrer suffisamment de temps aux travaux demandés? l'équipement en ordinateur personnel performant est-il suffisant; des difficultés d'accès au réseau se pose-t-il?

- Pratiques d'étude: Quels usages les étudiants font-ils des supports et des outils de communication mis à disposition? Les utilisent-ils comme leurs concepteurs l'imaginent? Les exercices, activités ou travaux pratiques proposés en ligne favorisent-ils un travail indépendant efficient chez les étudiants?

- Connaissances acquises: Les objectifs d'apprentissage sont-ils atteints? Les compétences développées correspondent-elles à celles attendues? Des savoirs et savoir-faire non prévus par les auteurs du cours sont-ils acquis?

Sur le plan méthodologique, plusieurs modalités de recueil de données ont été adoptées: entretiens avec les enseignants concernés et avec des étudiants; enquêtes par questionnaires auprès des étudiants; observations directes de leçons; analyse de la communication médiatisée sur les forums; repérage des difficultés de compréhension rencontrées par les étudiants; analyse de leurs résultats finaux aux examens (Perret \& Schubauer-Leoni, 2002)

\section{Un cadre d'analyse}

Pour notre analyse, nous allons utiliser comme référence théorique certaines approches de la théorie des organisations (voir Pfeffer, 1997 et Scott, 2003 pour une revue).

En effet, les projets CVS possèdent les caractéristiques principales qui définissent une petite organisation (Scott, 2003, p. 25-30): ils ont un objectif défini (la réalisation de cours eLearning), une structure interne (avec un chef de projet clairement identifié et une répartition des tâches entre professeurs et assistants ou techniciens) et ils sont institutionnellement reconnus en tant que projets du CVS.

En tant que tels, les projets peuvent être considérés comme des acteurs sociaux, qui sont capables de poursuivre leurs objectifs et de faire des choix entre différentes alternatives. Cette capacité n'est toutefois pas absolue, mais elle est limitée par leur environnement. Il est utile de distinguer analytiquement entre deux dimensions environnementales (Scott, 2003, p. 133):

- la dimension matérielle: les ressources financières disponibles pour les projets, les personnes et leurs compétences, les instruments techniques, les infrastructures;

- la dimension institutionnelle 2 : l'ensemble des conceptions et des règles qui régissent le comportement des projets: il s'agit des conceptions spécifiques à 
l'eLearning (par exemple les modèles proposés par le CVS), mais aussi des règles et des aspects culturels généraux sur le système universitaire (la définition du rôle du professeur; les règles de décision dans les universités; celles pour les carrières académiques, etc.).

Une des distinctions principales dans la théorie des organisations concerne la mesure dans laquelle les organisations individuelles sont capables de faire des choix et de gérer activement leurs relations à l'environnement de manière à atteindre leurs buts. Des théories comme la gestion stratégique (Porter, 1980), l'économie institutionnelle (Moe, 1984; Williamson, 1973) ou la resource dependency theory (Pfeffer \& Salancik, 1978) soulignent la capacité des acteurs de décider rationnellement et conçoivent l'environnement comme un ensemble de contraintes et d'opportunités pour atteindre leurs buts. A l'encontre, une tradition qui trouve son origine dans les travaux pionniers de Herbert Simon (March \& Simon, 1958) a montré les limites cognitives de la rationalité et comment les modèles culturels influencent (a un niveau souvent non conscient) la vision du monde des acteurs et leurs choix. Le néoinstitutionnalisme a accentué la capacité des institutions à déterminer le fonctionnement des organisations (Powell \& DiMaggio, 1991; Scott, 2001, p. 75) et, notamment, l'importance de la dimension cognitive dans le comportement organisationnel (DiMaggio et Powell, 1983; Meyer \& Rowan, 1977). Le comportement des organisations n'est ainsi pas basé sur une évaluation rationnelle des différentes options, mais est largement dicté par des symboles et par des représentations implicites et suit souvent des procédures et des routines établies a priori.

Plutôt que de s'exclure, ces deux traditions présentent deux dimensions largement complémentaires du comportement de tout acteur social et c'est essentiellement un problème empirique de déterminer lequel des deux aspects - la capacité d'agir de manière stratégique ou la conformité au modèles institutionnalisés - joue le rôle le plus important (Scott et al., 2000, p. 67).

\section{Des objectifs et des représentations différentes}

L'environnement des projets CVS est constitué essentiellement par d'autres acteurs sociaux, comme les autorités politiques et les organisations de politique universitaire (Conférence Universitaire Suisse et Conférence des Recteurs des Universités Suisses), les organes du programme lui-même (comité directeur et secrétariat) et les organes que constituent les hautes écoles. Il s'agit ici d'examiner les représentations et les objectifs de ces acteurs par rapport à l'introduction de l'eLearning, ainsi que leur influence sur le développement même des projets. 


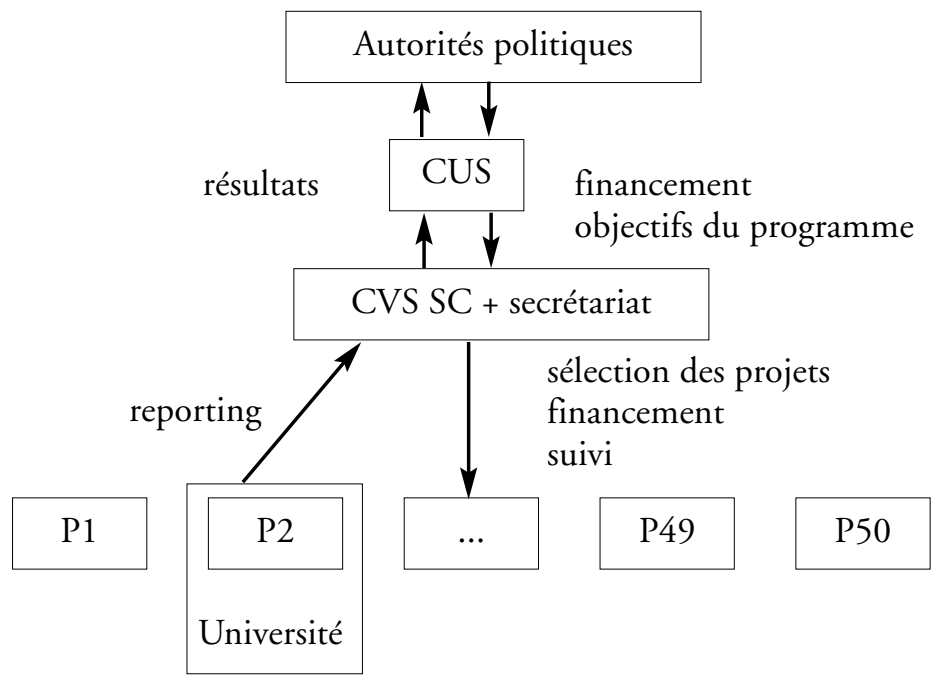

Figure 1: L'environnement des projets CVS

\section{Les autorités politiques}

Les autorités politiques (Conseil fédéral, parlement, Groupement pour la science et la recherche) ont défini dans le cadre du message FRT 2000-2003 (Conseil fédéral, 1998) les objectifs et le cadre financier pour le CVS. Le message FRT montre que le CVS est considéré comme un élément de la modernisation du système universitaire suisse. Cette origine conditionne les objectifs du programme, avec l'accent sur l'amélioration de la qualité de la formation de base et sur la coopération entre les universités, et surtout son insertion dans le contexte de la nouvelle Conférence Universitaire Suisse (CUS), qui était chargée en même temps de la coordination du système universitaire en Suisse. D'ailleurs c'est au sein de la CUS que les travaux préparatoires pour le CVS ont été menés, dans le cadre du groupe de travail Formation Universitaire et Nouvelles Technologies (FUNT; CUS, 1996, 1997).

Les autorités politiques définissent (ou légitiment) ainsi certains choix du programme et contrôlent le financement; ils délèguent toutefois complètement son exécution au CVS et aux hautes écoles. Ce sont notamment le CVS et la CUS qui sont appelés à rendre compte des résultats du programme, en vue aussi de sa prolongation éventuelle.

\section{Les hautes écoles}

Selon nos interviews, les hautes écoles sont beaucoup plus réticentes sur le potentiel de l'eLearning, qui est conçu comme un instrument pour améliorer l'enseignement dans le cadre actuel; avec une priorité aux disciplines qui concernent un 
grand nombre d'étudiants, notamment dans les sciences humaines et sociales (Lepori \& Succi, 2003). La plupart des universités sont aussi préoccupées par les coûts supplémentaires du eLearning et estiment qu'un appui de la Confédération est indispensable. Cette approche prudente caractérise la plupart des universités européennes (Collis \& van der Wende, 2002; van der Wende \& van de Ven, 2003), même s'il y a quelques cas où l'eLearning est utilisé pour développer de nouvelles offres éducationnelles (Lepori \& Succi, 2003). L'écart entre la rhétorique des NTIC au niveau politique et la pratique prudente des organisations d'enseignement supérieur a d'ailleurs été remarqué pour d'autres pays européens (Maassen \& Stensaker, 2003).

Les hautes écoles sont l'acteur le plus important pour les projets CVS: elles définissent leur environnement normatif, mais elles contrôlent aussi l'intégration de l'eLearning dans les cursus d'études (au niveau des départements et des instituts), les ressources propres, très souvent aussi le choix des collaborateurs et surtout leur future carrière.

\section{Le Campus Virtuel Suisse}

Le Campus Virtuel Suisse est composé par un secrétariat et par le comité directeur, c'est-à-dire l'organe qui est responsable de la sélection et du suivi des projets. La composition du comité directeur du programme (en avril 2004) est révélatrice de son orientation: sur huit membres, six sont des professeurs universitaires en informatique, un vient de l'Open University et le dernier d'une grande entreprise. Ainsi, dans ses documents officiels et dans son appel d'offres, le CVS préconise comme modèle pour l'eLearning la réalisation de cours à distance sur Internet, selon le modèle de certaines universités américaines; il développe des idées comme celle de «campus virtuel» et du remplacement de l'enseignement en présence, qui vont jouer un rôle important pour l'acceptation politique du programme.

L'impact sur les projets est fondamental dès leur phase de conception et jusqu'au au moment de leur sélection. En effet, les projets présentent des caractéristiques communes, puisqu'ils sont sélectionnés en fonctions de critères établis et incorporent dès le début certaines visées du programme. Les chefs de projets sont tous des professeurs des hautes écoles suisses. Les projets concernent surtout des cours de base avec un grand nombre d'étudiants et un des objectifs déclarés est la réalisation d'un cours pouvant être suivi à distance. Pendant la réalisation, le CVS dispose par contre de peu de moyens d'intervention, sinon par une pression normative surtout dans le cas le plus évident des projets qui ne respectent pas les délais. En termes organisationnels, il s'agit d'une relation entre un principal (le CVS) qui transfert des ressources à une agence (les projets) pour réaliser un objectif donné (introduire le eLearning dans les hautes écoles). Cette forme de délégation comporte toujours le risque que l'agence, après avoir été sélectionnée, s'attache à atteindre ses propres objectifs plutôt que ceux du principal (Coleman, 1990). 


\section{Un environnement contradictoire...et des espaces \\ de liberté}

La discussion précédente permet de cerner quelques aspects importants de la relation entre les projets CVS et leur environnement, et de formuler ainsi des hypothèses à vérifier empiriquement.

Premièrement, les projets dépendent d'autres organisations pour leur fonctionnement. En effet, il ne disposent pas de ressources propres, mais reçoivent les financements du CVS et des universités; l'engagement de collaborateurs dépend aussi de décisions des universités, ainsi que la mise à disposition de supports techniques. Ainsi, pour réussir dans leur entreprise, les responsables de projets sont appelés à développer des stratégies pour garder le soutien de ces organisations. De plus, ces acteurs - notamment le CVS et les universités - ont des perspectives différentes, voire contradictoires sur le eLearning. En cas de contradictions, les projets tendront à satisfaire les exigences des acteurs qui ont davantage de pouvoir (resource dependency theory; Pfeffer \& Salanzick, 1978). Dans notre cas, il s'agit des universités, puisque le CVS se limite à sélectionner et à financer les projets. Au delà de cette tâche qui caractérise un programme d'impulsion, le CVS n'a que peu de possibilités d'intervention et de contrôle. Ainsi, la première hypothèse est que les projets vont répondre surtout aux exigences de leurs universités d'appartenance plutôt qu'à celles du CVS.

Deuxièmement, pour être reconnus, les projets doivent répondre à certains critères qui les qualifient comme des projets CVS. Le CVS, en tant que programme de la Confédération pour développer le eLearning, jouit d'une légitimation forte, qui donne une force normative à ses conceptions même si après la sélection des projets, l'influence directe sur les projets est limitée. Ces critères comprennent notamment la production d'un matériel de cours sur Internet, des temps d'autoformation en partie à distance, la possibilité d'une utilisation en ligne complètement à distance, ceci par différentes universités. Ainsi, la deuxième hypothèse est que les projets ne sont pas libres de redéfinir arbitrairement ce qu'est le eLearning, mais doivent au moins partiellement se tenir aux définitions données par le CVS.

Troisièmement, les responsables de projet ont des intérêts et exigences spécifiques. Nos interviews montrent que dans la plupart des cas ils sont intéressés à innover dans l'activité didactique et à réaliser des cours plus stimulants pour les étudiants (Lepori \& Rezzonico, 2003). Dans une minorité des cas, de nouvelles exigences pratiques sont présentes (par exemple pouvoir offrir un cours dans plusieurs universités). L'objectif prioritaire des chefs de projet est ainsi une recherche de solutions aux besoins rencontrés dans leur propre activité d'enseignement, cela dans le cadre d'un modèle universitaire centré sur le professeur et son rôle à la fois d'enseignant et de chercheur. Notre troisième hypothèse est ainsi que les responsables des projets vont essayer de renégocier le modèle proposé par le CVS de manière à le rendre plus compatible avec leurs objectifs. 


\section{Effets collectifs et apprentissage institutionnel}

Jusqu'ici, nous avons considéré la relation entre les projets et leur environnement de manière unidirectionnelle: ainsi, les choix des projets sont limités et influencés par l'environnement, mais il n'ont pas la capacité d'influer sur les choix et le comportement d'acteurs comme les hautes écoles et les CVS. Cette assomption est justifiée pour des aspects généraux, comme le modèle et l'organisation du système universitaire, puisque les projets CVS sont (au moins actuellement) trop peu nombreux et petits pour changer ces données de fond. Toutefois, elle ne l'est pas pour des questions spécifiques à l'eLearning, puisque les projets CVS représentent en effet la première tentative d'introduire à grande échelle l'eLearning dans les hautes écoles suisses et, ainsi, il faut admettre la possibilité que leurs choix et expériences influencent les conceptions en la matière tant des hautes écoles que du CVS.

Cette possibilité est renforcée par le fait que nous sommes en présence d'une population de projets, qui ont la possibilité d'échanger entre eux des expériences, notamment dans le cadre des rencontres périodiques organisées par le CVS. Ainsi, pour garder sa force normative, le modèle de eLearning proposé par le CVS doit être adopté et reconnu par la plupart des projets: on verra cependant que dès qu'une majorité des projets s'oriente vers un modèle différent (celui du blended learning), ce modèle va devenir la référence pour l'ensemble du programme.

Ces effets d'apprentissage ne sont pas automatiques, mais dépendent à leur tour des choix des acteurs concernés et de leur environnement; ainsi, le CVS n'est pas non plus libre vis-à-vis des autorités politiques de modifier de manière arbitraire les objectifs du programme et ses modalités de réalisation, faute de perdre sa légitimité et la possibilité de financements futurs. En plus, comme tout autre organisation, un programme peut avoir des capacités limitées (mais variables selon le sujet et la situation) de modifier ses conceptions et son mode de fonctionnement («inertie organisationnelle»; Hannan \& Freeman, 1989).

Notre quatrième hypothèse est ainsi qu'il y a la possibilité d'effets d'apprentissage au niveau du programme dans son ensemble, surtout si la plupart des projets font les mêmes choix, mais qu'elle doit être examinée cas par cas, ce que nous allons faire dans la suite de cet article.

\section{Les choix opérés au sein des projets et l'apprentissage institutionnel}

Dans cette section, nous allons prendre appui sur le cadre théorique présenté précédemment pour analyser les choix effectués par les équipes de projets. Les trois dimensions que nous retenons ici présentent un caractère exemplaire, elles révèlent d'une part des enjeux centraux pour les projets et elles ont d'autre part été largement débattues au sein du CVS. Elles concernent: 
- la relation entre les temps d'activités en présence et les temps d'apprentissage à distance par Internet;

- le choix du support technologique;

- le développement de matériaux de cours réutilisables et leur adaptation aux différents contextes d'enseignement.

L'analyse de chacun de ces points est divisée en trois parties. D'abord, nous présentons les conceptions de départ au niveau institutionnel (CVS et hautes écoles); ensuite, nous analysons les pratiques effectives mises en oeuvre dans les projets, pour finalement examiner dans quelle mesure ces pratiques ont entraîné une révision des objectifs et des stratégies au niveau du CVS et des universités.

\section{Quelle mise à distance préconiser?}

\section{Les positions en jeu}

Dans sa mise à concours de 1999, le CVS propose de développer des cours complètement en ligne qui seraient suivis par les étudiants des universités suisses indépendamment de leur localisation. Le modèle de l'enseignement à distance est ainsi indirectement adopté, même s'il concerne les universités conventionnelles existantes. La mise à distance préconisée reste toutefois parcellaire à l'échelle de cours isolés, puisque l'option du développement de cursus complets n'a pas été retenue. Le modèle adopté est cohérent avec l'objectif de développer des cours «à la carte»: il s'agit de mettre à disposition des modules d'enseignement de haute qualité et largement réutilisables; la dimension du marché potentiel permettrait de récupérer l'investissement initial.

Les concepts et les priorités des universités sont par contre différents. La mise à distance est perçue souvent comme un risque pour la qualité de l'enseignement et la priorité est donnée à une amélioration de l'enseignement pour les étudiants locaux, notamment pour les cours avec un effectif élevé d'étudiants (Lepori \& Succi, 2003).

C'est dans cette perspective que s'engagent les professeurs intéressés. La mise à distance envisagée est partielle et n'est plus géographique, elle consiste à réduire le nombre d'heures d'enseignement au profit du travail autonome des étudiants. Mais l'intention des promoteurs du CVS n'est pas pour autant oubliée, d'où la tension observée dans la conception même des démarches d'enseignement mise en oeuvre.

\section{Les choix des projets}

Le constat principal est que la plupart des projets n'ont pas réalisé des cours complètement à distance. Sur 18 projets analysés, quatre d'entre eux visent une offre de formation entièrement à distance, tandis que les 14 autres ont choisi un modèle hybride (Lepori \& Rezzonico, 2003). Nous avons pu cerner trois modèles principaux qui se caractérisent par:

- la substitution de quelques heures de cours en présence par un temps d'autoformation, et ceci spécialement pour les chapitres qui se prêtent le mieux au 
moyen électronique (lorsque interviennent par exemple des représentations en 3D, des supports multimédia, des exercices interactifs, ...);

- l'utilisation de logiciels, pour les leçons ou les exercices, qui améliorent l'efficacité de l'apprentissage, qui permettent de gagner du temps et de personnaliser le travail des étudiants;

- l'utilisation du matériel de cours en ligne comme appoint pour les leçons en présence. Le contenu en ligne est utilisé comme outil pour améliorer le niveau des discussions en classe.

Le choix entre ces modèles dépend bien sûr des caractéristiques de la discipline concernée, mais aussi des conditions de départ; on observe par exemple que la mise à distance concerne surtout les projets qui développent de nouveaux cours.

L'analyse des réalisations montre une grande diversité dans les modalités d'intégration des temps de formation à distance et en présence (blended learning). Les recompositions des différents temps d'enseignement et d'apprentissage sont multiples. Pour rendre compte de cette diversité, nous avons recouru à une représentation schématique des dispositifs d'enseignement selon leur manière d'articuler trois composantes principales: les heures de cours, la communication médiatisée et le travail personnel (Schubauer-Leoni \& Perret, 2003). Les dispositifs d'enseignement représentés ci-dessous sont trois exemples de mise en oeuvre d'un module de cours dans le cadre des tests de terrains effectués.

Le premier dispositif se caractérise par une alternance régulière des heures de cours (exposés et discussion) et du travail personnel des étudiants, avec une possibilité de communication médiatisée pour toute question ou demande d'aide.

La figure 2 illustre une autre démarche: les heures de cours sont en partie transformées en séances d'études et d'exercices avec l'appui d'un assistant qui intervient à la demande. Ces séances se prolongent par un temps de travail individuel. Les heures d'enseignement proprement dit se limitent à un cours introductif pour présenter le module et à une séance conclusive.

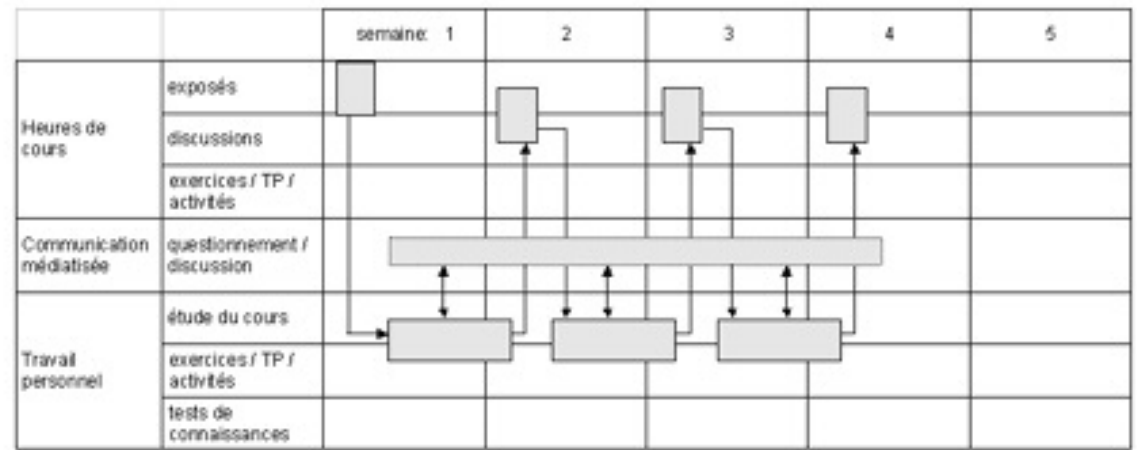

Figure 2: Alternance régulière des heures de cours et de l'étude personnelle 
Les figures 3 et 4 illustrent un dispositif plus différencié qui assignent à chaque période de cours ou de travail personnel une intention didactique spécifique.

\begin{tabular}{|c|c|c|c|c|c|c|c|c|c|c|}
\hline & & tensiax 1 & 2 & 3 & 4 & 5 & 6 & ? & 8 & ? \\
\hline \multirow{3}{*}{$\begin{array}{l}\text { Hewes on } \\
\text { chant }\end{array}$} & exposot & 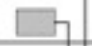 & & & & & & & त & \\
\hline & ascustion & & & & & & & & - & \\
\hline & actudsibueroces & & & & & & & & & \\
\hline 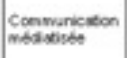 & $\begin{array}{l}\text { antionnements } \\
\text { iscuslion }\end{array}$ & & & & & & & & & \\
\hline \multirow{3}{*}{ Taver } & torite dis couns & & & & & & & & & \\
\hline & aturtingericest & & & & & & & & & \\
\hline & $\begin{array}{l}\text { potss de } \\
\text { octrnassances }\end{array}$ & & & & & & & -1 & & \\
\hline
\end{tabular}

Figure 3: Heures de cours transformées en séance d'exercice et d'étude

\begin{tabular}{|c|c|c|c|c|c|c|}
\hline & & semsine. 1 & 2 & 3 & 4 & 5 \\
\hline \multirow{3}{*}{$\begin{array}{l}\text { Heures de } \\
\text { cours }\end{array}$} & exposts & & & & & \\
\hline & diecussions & & & & & \\
\hline & $\begin{array}{l}\text { exercikes s TP f } \\
\text { activtes }\end{array}$ & & & & & \\
\hline $\begin{array}{l}\text { Communicasion } \\
\text { medatses }\end{array}$ & $\begin{array}{l}\text { questioenement } s \\
\text { discussion }\end{array}$ & & & & & \\
\hline \multirow{3}{*}{$\begin{array}{l}\text { Traval } \\
\text { pessonnel }\end{array}$} & étude du cours & & & & & \\
\hline & $\begin{array}{l}\text { exercices S TPi i } \\
\text { actortes }\end{array}$ & & & & & \\
\hline & $\begin{array}{l}\text { tests de } \\
\text { cornassancestes }\end{array}$ & & & & & \\
\hline
\end{tabular}

Figure 4: Articulation fine des différents temps pédagogiques

La diversité des démarches mises en œuvre est très grande, nous pourrions en donner de multiples autres exemples. La palette des réalisations se révèle en effet beaucoup plus large que les solutions initialement imaginées ou préconisées au sein des projets. En effet, les décisions en jeu ne se limitent pas à déterminer le pourcentage des heures de cours à maintenir, mais à gérer un ensemble de décisions qui en découlent. Des solutions sont à inventer: quelles heures de cours maintenir? dans quel but précis? en amont ou en aval du travail personnel des étudiants? Les enseignants sont susceptibles d'apporter des réponses différentes selon leur propre expérience de l'enseignement, le nombre d'étudiants concernés, les possibilités d'encadrement, ou encore selon les contraintes d'horaires et de salles. 


\section{Les implications institutionnelles et une tentative de bilan}

Au niveau des projets, la tension observée au démarrage des projets concernant la part de ce qui allait être mis ou non à distance, a conduit à une évolution de la réflexion. La question centrale n'est plus vraiment aujourd'hui de savoir si le cours est à concevoir entièrement ou partiellement à distance, mais de trouver les meilleures articulations possibles entre les temps didactiques individuels et collectifs, ainsi qu'entre les temps de communication présentielle et médiatisée. De telles recompositions s'avèrent complexes à gérer en raison des nombreux facteurs psychologiques, didactiques organisationnels et institutionnels chaque fois en jeu. La variété des formes que peut prendre le déroulement d'un cours, est probablement un indicateur d'adaptabilité susceptible de garantir la pérennité d'un projet.

Au niveau institutionnel, le bilan est plus contrasté. Le CVS a accepté dans la pratique les modèles hybrides, même si cela a occasionné des discussions au moment de l'évaluation de certains projets. Dans son rapport 2001 à l'Office fédéral de l'éducation et de la science le CVS affirme que «il est apparu que l'objectif de réaliser des cours complètement en ligne ne correspond pas aux besoins des étudiants dans les curricula de base et que, en général, le contact personnel (au moins sporadique) est indispensable dans ces cours. L'approche courante est aujourd'hui celle du 'blended learning' qui combine l'enseignement en présence et à distance» (traduction libre de l'allemand par les auteurs).

Toutefois, le programme de consolidation (2004-2007) et la nouvelle mise au concours d'une série de projets lancés par le CVS confirment la diminution de l'enseignement en présence comme un des objectifs principaux du programme. En même temps, l'élaboration de scénarios didactiques plus élaborés est explicitement demandée pour les nouveaux projets.

Ainsi, au gré des expériences faites, il s'est produit une acceptation générale d'un modèle qui prévoit des formes de eLearning adaptées à chaque contexte d'usage et liées à l'élaboration de scénarios didactiques détaillés. Cette évolution se caractérise par une complexification du modèle préconisé initialement par le CVS. L'évolution apparaît par contre plus limitée au niveau de la conception générale de l'offre de cours. En effet, le CVS confirme le modèle basé sur le développement de cours isolés et accentue probablement leur standardisation en attribuant leur réalisation technique aux centres de support des hautes écoles.

\section{Conception du matériel de cours Les positions en jeu}

En préconisant le développement de cours qui puisse être suivi entièrement à distance, les promoteurs du CVS prennent implicitement comme modèle de référence les matériels de cours développés pour l'enseignement à distance. Ce matériel se caractérise généralement par la mise à disposition: d'un texte complet de référence enrichi par différents médias complémentaires; d'une série d'activités proposées aux étudiants, ainsi que des indications méthodologiques détaillées sur 
la manière de planifier et de réaliser le travail d'étude attendu (Rowntree, 1994).

Ce modèle, fruit d'une longue expérience au sein des institutions d'enseignement à distance se trouve assez éloigné des pratiques habituelles de communication didactique dans l'enseignement universitaire conventionnel. Il convient cependant de prendre ici en compte les traditions et les pratiques propres à chaque domaine d'enseignement. La référence à un manuel ne joue pas le même rôle selon les disciplines enseignées. Si un tel matériel de cours remplit une fonction pédagogique clé dans certains domaines, dans d'autres (en particulier dans les sciences humaines et sociales) le matériel de cours est généralement conçu comme un ensemble de ressources diverses et multiples que les étudiants sont appelés à chercher en bibliothèque, à sélectionner et à étudier. Ceci est à mettre en relation avec les pratiques de lecture qui varient selon les formations suivies par les étudiants (Fraisse, 1993; Lahire, 1997).

La diversité des cultures didactiques et pédagogiques concernant les supports d'études et les manières d'apprendre à l'université reste un domaine encore en grande partie à explorer (Frenay et al., 1998). La conception même des supports de cours électroniques ne peut ignorer le rapport que professeurs et étudiants entretiennent classiquement avec les textes et les activités d'étude propres à leurs disciplines. De fait, la référence implicite des promoteurs du CVS semble être surtout celle des formations scientifiques pour lesquelles le recours à un manuel comme support principal de cours et à des séries d'exercices est une pratique fréquente. Même lorsque le principe d'utiliser un matériel de cours déterminé est acquis, son usage effectif peut encore varier. Nous avons constaté à plusieurs reprises l'importance pour les enseignants de pouvoir disposer d'un matériel modulable, configurable à leurs propres objectifs et à leur contexte d'enseignement.

\section{Les choix des projets}

Il apparaît que la plupart des projets ont investi une grande partie de leurs ressources dans le développement de matériels mis sur Internet. Le coût élevé des projets est ainsi essentiellement lié aux investissements pour produire de nouveaux supports et pour les mettre dans une forme adaptée au WWW. Selon nos interviews, le modèle prévalent n'est pas celui du cours unitaire, mais plutôt d'une collection de modules qui gardent une certaine autonomie dans leur conception et leur contenu. Les projets varient dans le degré d'uniformité des modules: dans certains cas, les modules suivent un plan et une présentation uniforme, dans d'autres cas ils se présentent comme des éléments de cours quasiment indépendants.

En plus des caractéristiques liées au domaine scientifique concerné, les choix effectués dépendent aussi de l'organisation de la réalisation: les projets plus centralisés, où la leading house développe l'essentiel du contenu, produisent des modules plus uniformes que les projets où participent différents instituts. L'idée de produire des cours unitaires, de conception homogène, se révèle ainsi difficilement compatible avec celle de favoriser la coopération interuniversitaire. 
Une source d'ambiguïté est liée aux scénarios d'usage envisageables. Une interrogation fréquemment soulevée par les enseignants engagés dans le développement d'un cours CVS peut se résumer ainsi: faut-il concevoir un matériel de cours sur support électronique qui puisse convenir à son propre enseignement, avec les étudiants présents? Ou faut-il penser à son utilisation «hors site» par un public d'étudiants distants (supposés intéressés, mais souvent non encore identifiés)? Dans chaque cas de figure, les enseignants perçoivent bien que ce ne sont pas exactement les mêmes démarches de formation qui sont en jeu. En particulier, les contraintes relatives à la rédaction du matériel de cours ne sont pas les mêmes si ce matériel doit pouvoir être compris et travaillé par l'étudiant en toute autonomie, ou selon qu'il peut faire l'objet de présentations orales complémentaires, ou de discussions lors de cours ou de séminaires. La mise en forme des exercices ou l'explicitation des activités d'apprentissage ne prennent pas la même tournure selon les types d'accompagnement (tutoring) adoptés. De même, les textes d'introduction ou de liaison entre les chapitres d'un cours ne prennent pas la même importance et le même sens, si certaines heures de cours sont maintenues ou non pour expliciter les visées, les attentes et assurer «l'avancement» du cours.

\section{Les implications institutionnelles et une tentative de bilan}

Les deux perspectives en jeu se sont révélées difficiles à articuler. L'intérêt des professeurs pour un matériel de cours qui se prête à divers usages, en quelque sorte à la carte, a pu être perçu comme une solution de retrait par rapport à la perspective du CVS de diffuser voire de commercialiser une offre de cours complet, susceptible d'être livrés à distance «clé en main».

En effet, la tâche de développer un produit relativement stable et diffusable (tâche apparenté au travail d'édition d'un ouvrage) apparaît d'une autre nature que celle de l'enseignant qui réunit le matériel nécessaire pour faire «vivre» son cours au fil des semaines. Les deux tâches répondent aussi à des modèles de gestion économiques très différents; ce n'est pas un hasard si classiquement, la production de manuels est confiée à des éditeurs spécialisés (qui disposent des ressources et du marché pour amortir les coûts de réalisation et de réédition).

\section{Les choix technologiques}

\section{Les positions en jeu}

Concernant le choix de l'instrument technologique différentes options sont en présence. Pour un ensemble de raisons économiques et d'interopérabilité le CVS en est venu à préconiser le choix d'une plate-forme standard. Pour ce faire, les plates-formes existantes ont été comparées afin de mieux connaître les caractéristiques de chacune, leurs fonctionnalités, leurs points forts et leurs points faibles. Ce processus d'évaluation a conduit le CVS à proposer l'utilisation de la plateforme WebCT. 
Au sein des Universités, les choix technologiques ont été laissés à la charge des professeurs engagés dans un projet CVS. Les Universités gèrent certes les infrastructures informatiques nécessaires aux activités de recherche, de communication et d'administration. Mais le choix de supports d'enseignement spécifiques, telle une plate-forme de eLearning, relève de la responsabilité des enseignants concernés et, à l'exception de Saint Gall, aucune autre université suisse a fait le choix d'une plate-forme unique pour l'ensemble des projets de eLearning.

\section{Les choix des projets}

Dans la pratique, environ la moitié des projets CVS ont opté pour WebCT, tandis que les autres ont produit eux-mêmes une solution technologique pour répondre à leurs propres besoins. Les débats sur les avantages et désavantages de telle ou telle option sont parfois tendus. De toute évidence, ces choix ne sont pas que technologiques, des préoccupations organisationnelles et pédagogiques soustendent le débat.

La première préoccupation porte sur le niveau de complexité du système adopté, les compétences informatiques qu'il requiert et le degré de contrôle possible du système. Les enseignants non experts en informatique craignent que l'outil choisi exige l'appui permanent d'un informaticien, et que cette collaboration vienne alourdir le fonctionnement de leur institut. Leur aspiration est de pouvoir gérer une plate-forme et des supports simple d'usage. Notons que cette question ne se pose pas de la même manière lorsque le projet s'inscrit dans un institut où les compétences informatiques sont déjà bien présentes. Dans ce cas, on observe une réticence des équipes de projet à l'égard des plates-formes commerciales perçues comme trop rigides par rapport aux outils que ces équipes ont la possibilité de développer et d'adapter par elles-mêmes.

La deuxième préoccupation a plus directement trait à la manière de concevoir le déroulement d'un enseignement et le rôle du professeur. En effet, l'enseignement à distance a donné lieu à une redéfinition du rôle des professeurs dont la charge essentielle, à titre de professeur-auteur, est de rédiger un matériel de cours, avec toutes les indications méthodologiques qui permettent à l'étudiant à distance de planifier son étude. Il s'agit alors de préparer à l'avance l'ensemble du matériel de cours mis à disposition des étudiants, avec un planning précis de lecture et d'exercices à effectuer régulièrement.

Par contre, dans le cadre universitaire conventionnel, l'activité d'enseignement comprend deux tâches complémentaires: celle de préparer certes des supports de cours, mais aussi celle de "faire vivre» la matière du cours en situation de communication orale, ce qui impose une certaine adaptabilité du déroulement. En effet, lorsque les circonstances, les difficultés rencontrées ou les questions posées par les étudiants l'exigent, le plan initial du cours est souvent ajusté, des approfondissements ou des indications complémentaires sont donnés. Un matériel de cours déposé sur une plate forme standard se prête-t-il aisément à de telles adaptations? Le professeur-auteur en garde-t-il le contrôle, en reste-t-il maittre? 
Les systèmes d'aide à l'édition du matériel de cours apportent-ils une réponse adéquate (Burdet, Muttoni \& Perret, 2002)? Quels sont les coûts des adaptations régulières, voire de refonte du contenu du cours à plus long terme?

Finalement, une dernière préoccupation concerne les ressources (humaines et financières) nécessaires pour maintenir des outils technologiques autoproduits, une préoccupation très présente dans les services informatiques des universités. La logique d'une réduction des coûts de production et de maintien par une certaine standardisation s'accommode ainsi difficilement des exigences de flexibilité et d'adaptation fine aux conditions d'enseignement.

\section{Les implications institutionnelles et une tentative de bilan}

Le CVS a de fait accepté que certains projets développent leur propre plateforme, mais n'a pas pour autant renoncé a poursuivre une standardisation. Il a ainsi financé l'installation d'une plateforme nationale WebCT qui sera disponible en 2004 et il vise la création d'une infrastructure d'authentification qui permette aux étudiants de différentes universités de s'inscrire aux cours CVS.

L'innovation principale a toutefois été de confier à des centres de support dans les hautes écoles le développement et la gestion technique des nouveaux projets. A cette fin, une partie des ressources du CVS est utilisée pour créer ou consolider ces centres. Par cette innovation, ce qui est visé est une professionnalisation de la production de cours et une réduction substantielle des coûts de production.

Toutefois, les centres de support sont confrontés aux mêmes questions que les projets de la première phase. S'ils choisissent de n'utiliser qu'une ou deux platesformes commerciales, ils répondront difficilement aux demandes des professeurs qui souhaitent la plus grande adaptabilité de leurs supports de cours. Inversement, une trop grande diversité pourrait entraîner des difficultés de gestion et surtout une augmentation des coûts. Selon nos interviews, la majorité des responsables des Centres ont une position pragmatique et préconisent une utilisation flexible des différents outils technologiques. La capacité réelle des Centres de support à définir les lignes de développement technologique va toutefois surtout dépendre de leur position et légitimité à l'intérieur des hautes écoles.

\section{Conclusions}

L'objectif de cet article n'était pas de proposer un modèle ou de donner des réponses aux maintes questions ouvertes sur le développement de l'eLearning en Suisse, mais plutôt de montrer la complexité des processus d'apprentissage organisationnel au sein du Campus Virtuel, et aussi leur rôle dans la diffusion du eLearning dans les hautes écoles suisses. Nous résumerons les principaux résultats obtenus en nous référant aux hypothèses formulées dans la première partie de l'article. 
Sollicités, il y a une dizaine d'années, sur l'avenir de l'enseignement à distance en Suisse, les responsables politiques et les groupes de travail qui ont oeuvré à promouvoir le programme CVS ont réagi en s'inspirant d'un modèle connu, celui de l'enseignement à distance, pour l'adapter au contexte institutionnel du système universitaire suisse. C'est au moment de la réalisation du programme, et en particulier lors de l'élaboration et de l'expérimentation des premiers modules de cours, qu'il est clairement apparu que ce modèle relevait d'une vision du fonctionnement de l'enseignement universitaire éloignée de celle actuellement dominante dans les universités suisses.

Dans ce contexte, presque tous les responsables de projets ont écarté ce qui dans ce modèle était difficilement compatible avec le fonctionnement habituel de l'enseignement universitaire, comme la suppression des heures de cours donnés par les professeurs (au profit du travail autonome des étudiants), ou la création de matériels de cours standards pour tous les étudiants intéressés par une offre de cours alternative, quelque soit leur université d'appartenance. Le cadre normatif et organisationnel des cours développés au sein du programme CVS reste ainsi celui des universités existantes. De ce point de vue, le choix initial de développer l'eLearning dans ces hautes écoles, au lieu de créer une institution ad hoc, a largement conditionné l'orientation générale des réalisations.

Toutefois, les responsables des projets n'ont pas complètement ignoré le modèle proposé par le CVS. Nous avons ainsi pu observer des recompositions originales entre le modèle initialement proposé et l'enseignement universitaire traditionnel, notamment en ce qui concerne l'articulation des temps didactiques en présence et à distance, ou encore pour le passage d'une structure de cours unitaire à une structure modulaire, avec une certaine différenciation des modules élaborés. Dans certains cas, les projets ont aussi choisi de découpler la conformité aux modèles institutionnalisés et leur fonctionnement concret (Meyer \& Rowan, 1977): ainsi, alors que dans les présentations officielles le développement d'un cours sur Internet est annoncé, ce sont en fait des supports à l'enseignement présentiel qui se trouvent développés. Les interviews montrent aussi que les options retenues dépendent largement des exigences et des situations spécifiques de chaque projet: ainsi par exemple, la décision d'adopter ou non une plate-forme standard est influencée par la présence ou l'absence de compétences informatiques dans l'institut qui gère le projet.

Face au modèle initialement proposé par le CVS et à sa relative incompatibilité avec le contexte universitaire actuel, la plupart des projets ont géré activement ces tensions pour réaliser des cours qui répondent davantage à leurs propres attentes. De plus, pour réaliser ces arbitrages, les responsables des projets ont de fait joué sur les rôles différents du CVS et des universités: en effet, pour s'intégrer dans les pratiques universitaires actuelles, les projets ont été conduits à se distancer du modèle du CVS, tandis que les financements obtenus leur ont donné une certaine autonomie par rapport aux universités pour renouveler certaines pratiques d'enseignement. La disparité des modèles en jeu a créé ainsi des 
marges de manœuvre pour l'adaptation des projets aux exigences et aux aspirations locales.

Finalement, nous avons pu cerner de quelle manière ces adaptations ont eu un impact institutionnel et au niveau de l'organisation du programme. Ainsi, la signification de certains concepts a évolué (celle de eLearning notamment) et on observe une plus grande capacité (au moins au niveau du discours) à tenir compte de la diversité des situations réelles d'enseignement. En plus, dans la phase de consolidation du programme qui a démarré en 2004, l'accent est davantage mis sur le développement de scénarios pédagogiques adaptés aux différents besoins et contextes d'enseignement. Finalement, au niveau organisationnel, la création de centres de support dans les hautes écoles, a été voulue justement pour essayer de concilier la diversité des projets avec une certaine standardisation et réduction des coûts de production.

Notre conclusion est que la gestion de l'écart entre les objectifs initiaux du Campus Virtuel Suisse et ce qui a été réalisé dans une première phase du programme constitue un indicateur d'adaptabilité et de capacité de gestion stratégique dont font preuve tant les chefs de projets que les responsables du programme lui-même. Un tel écart est probablement inévitable, le prendre sérieusement en compte permet, par adaptations successives, de rendre compatible avec le système universitaire suisse un modèle initial de l'eLearning inspiré de contextes d'enseignement universitaire très différents: celui des institutions d'enseignement à distance, mais aussi celui de l'enseignement supérieur aux Etats-Unis.

\section{Notes}

1 Nous utilisons le terme «eLearning» pour désigner de manière générique l'utilisation des nouvelles technologies de l'information et de la communication (NTIC) pour améliorer la qualité de l'apprentissage, faciliter l'accès à des ressources et des services et l'échange et la collaboration à distance (CEC, 2001,2). Ce terme recouvre ainsi des applications très différentes du simple support aux cours en présence jusqu’à des cours pouvant être suivis entièrement par Internet (Bates, 2001). Par ailleurs, le terme n'assume pas à priori que l'introduction des NTIC implique une modification des méthodes d'enseignement et des pratiques d'apprentissage.

2 De manière générale, on peut définir une institution comme une structure sociale avec un certain degré de stabilité, qui définit des règles pour le comportement des acteurs (Scott, 2001, 48ss). Les institutions comprennent ainsi les systèmes de régulation (par exemple les normes juridiques ou les règles d'organisation et les procédures), avec une force coercitive sur le comportement des acteurs, les valeurs et les normes, qui définissent ce que les acteurs devraient faire et la manière de le réaliser, ainsi que toute une série d'aspects culturels comme les symboles ou les représentations de la réalité.

3 Toutes les références en ligne ont été vérifiées le 30 septembre 2004. 


\section{Références ${ }^{3}$}

Bates, T. (2001). National strategies for eLearning in post-secondary education and training, UNESCO. Paris: International Institute for Educational Planning [http://unesdoc.unesco. org/images/0012/001262/126230e.pdf].

Burdet, O., Muttoni, A. \& Perret, J.-F. (2002). Author-centered authoring: a necessity, Lausanne: EPFL

CEC (2001). Communication from the Commission to the council and the European Parliament, The eLearning Action Plan: Designing tomorrow's education. Brussels: COM (2001) 172, 28.3.2001.

Coleman, J.S. (1990). Foundations of Social Theory, Cambridge, Mass.: Harward University Press.

Collis, B. \& Van der Wende, M. (2002). Models of technology and Change in Higher Education. CHEPS, Toegepaste Onderwijskunde [http://www.utwente.nl/cheps]

Conseil fédéral (1998). Message concernant l'encouragement de la formation, de la recherche et de la technologie pour la période 2000-2003. Berne.

CUS (1996). Enseignement à distance au niveau universitaire. Rapport du groupe de travail de la Commission de Planification Universitaire. Berne: CUS.

CUS (1997). Création d'un Campus Virtuel Suisse, Rapport du Groupe d'experts «Formation universitaire et nouvelles technologies» de la Commission de Planification Universitaire. Berne: CUS.

DiMaggio, P. \& Powell, W. (1983). The Iron Cage Revisited: Institutional Isomorphism and Collective Rationality in Organisational Fields. American Sociological Review, 48, 147-160.

Frenay, M., Noël, B., Parmentier, Ph. \& Romainville, M. (1998). L'étudiant-apprenant: grilles de lecture pour l'enseignant universitaire. Bruxelles: De Boeck, Université.

Fraisse, E. (1993), Les étudiants et la lecture. Paris: Presses Universitaires de France.

Gurtner, J.-L., Monbaron, J., Platteux, H., Touvet, D. \& Zahnd, J. (2004). Dynamique de l'évolution des institutions de formation tertiaire suscitée par l'introduction des TIC. Synthèse du Programme National de Recherche 43. Berne [http://www.nfp43.unibe.ch/documentation/synthesis.htm].

Hannan, M. T. \& Freeman, J. (1989). Organizational Ecology. Cambridge, Mass.: Harward University Press.

Lahire, B. (1997). Les manières d'étudier. Paris: La Documentation Française.

Lepori, B., Cantoni, L. \& Succi, C. (2003). The introduction of eLearning in European universities: models and strategies. M. Kerres \& B. Voss (Hrsg.), Digitaler Campus. Vom Medienprojekt zum Nachhaltigen Medieneinsatz in der Hochschule. Münster: Waxmann, [www.edum.ch]

Lepori, B. \& Rezzonico, S. (2003). La realisation de cours Learning. Le cas du Campus Virtuel Suisse. 1st EDUM report. Lugano [www.edum.ch].

Lepori, B. \& Succi, C. (2003). E-Learning in Higher Education. Prospects for Swiss Universities, 2nd EDUM report. Lugano [www.edum.ch].

Lepori, B. \& Succi, C. (2004). eLearning in the Universities for Applied Sciences, Lugano, [www.edum.ch].

Maassen, P. \& Stensaker, B. (2003). Loosely Coupled Policy Links - ICT Policies and Institutional Dynamics in Norwegian Higher Education In M. Wende van der \& M. Ven van de (ed). (pp. 51-74.) In Wende \& Ven (2003): 51-74.

March, J. \& Simon, H. (1958). Organizations. New York:Wiley.

Meyer, J. \& Rowan, B. (1977). Institutionalized Organizations: Formal Structures as Myth and Ceremony. American Journal of Sociology, 83, 340-363.

Moe, T. (1984). The New Economics of Organization. American Journal of Political Science, 28, (4), 739-777. 
Perret, J.-F. (2004). Evaluer la production d'un cours en ligne; le projet I-Structure. Documents de recherche. Lugano: Università della Svizzera italiana, Istituto Comunicazione e Formazione.

Perret, J.-F. \& Schubauer-Leoni, M.-L. (2002). Evaluation pédagogique du projet SwisslingCVS. Test du module prototype «Semiotica verbale». Documents de recherche. Lugano: Università della Svizzera italiana, Istituto Comunicazione e Formazione.

Perret, J.-F. \& Schubauer-Leoni, M.-L. (2004). Swissling: un cours de linguistique en ligne. Synthèse d'une évaluation. Documents de recherche. Lugano: Università della Svizzera italiana, Istituto Comunicazione e Formazione.

Pfeffer, J. (1997). New Directions for Organization Theory, Problems and Prospects. Oxford: Oxford University Press.

Pfeffer J. \& Salancik, G. R. (1978). The External Control of Organizations: A Resource Dependence Perspective. New York: Harper and Row.

Porter, M. (1980). Competitive Strategies: Techniques for Analyzing Industries and Competitors. New York: Free Press.

Powell, W. \& DiMaggio, P. (1991). The New Institutionalism in Organizational Analysis. Chicago and London: The University of Chicago Press.

Rowntree, D. (1994). Preparing materials for Open, Distance and Flexible Learning. London: Kogan Page.

Schubauer-Leoni, M. \& Perret, J.-F. (2003), Dispositifs d'enseignement de la linguistique à l'Université. Communication aux rencontres du REF (Réseau Education et Formation). Genève, septembre 2003.

Scott, R.W. (2001). Institutions and Organizations. Thousands Oaks: Sage.

Scott, R.W. (2003). Organizations. Rational, Natural and Open Systems. Upper Saddle River: Pearson Education.

Scott, R.W., Ruef M., Mendel P. \& Caronna C. (2000). Institutional Change and Healthcare Organizations. Chicago: The University of Chicago Press.

Wende, M. van der \& Ven, M. van de (2003). The use of ICT in Higher Education. A mirror of Europe, Utrecht: LEMMA Publishers.

Williamson, O. E. (1975). Markets and Hierarchies. New York: The Free Press.

Mots clés: acquisition de connaissances, contrôle de la qualité, didactique, évaluation, méthode d'enseignement, ordinateur, orientation, technologie de l'information et de la communication, université 


\section{Institutionnelle Dynamiken und Projektstrategien im Swiss Virtual Campus}

\section{Zusammenfassung}

In diese Beitrag untersuchen wir die Entwicklung der Swiss Virtual Campus (SVC)-Projekte und ihre Wechselwirkung mit dem institutionellen Kontext. Wir zeigen auf, dass die Ansichten des SVC-Programms und der Universitäten darüber, was e-Learning ist und wie es eingeführt werden soll, divergieren. In diesem Zusammenhang waren die SVC-Projekte dazu geführt worden, vom ursprünglich vom SVC vorgeschlagenen Modell teilweise abzurücken zu Gunsten der Entwicklung von originalen Lösungen für jeden Einsatzbereich. Diese Erfahrungen führten zu einer Überarbeitung des e-Learning-Konzeptes auf Stufe Gesamtprogramm (in Richtung eines hybriden Lernansatzes) und bis zu einem gewissen Grad auch zu einer Änderung der Implementierungsstrategien durch die Einführung von universitären Support-Zentren. Abschliessend zeigen wir auf, dass zahlreiche Fragen noch immer offen sind, vor allem was die Organisation und die Finanzierung dieses e-Learning-Modells anbelangt.

Schlagworte: Beratung, Computer, Didaktik, Evaluation, Hochschule, Informations- und Kommunikationstechnologie, Lernen, Qualitätskontrolle, Unterrichtsmethode

\section{Campus Virtuale Svizzero. Le dinamiche istituzionali e le scelte dei responsabili dei progetti}

\section{Riassunto}

Questo articolo esamina lo sviluppo dei progetti del Campus Virtuale Svizzero (CVS) e la loro relazione con il contesto organizzativo e istituzionale. La nostra analisi mostra che il Campus Virtuale da una parte, le scuole universitarie svizzere dall'altra hanno delle concezioni molto diverse sul significato dell'eLearning e sulle sue modalità di introduzione nell'insegnamento universitario. In questo contesto, i progetti CVS si sono progressivamente distaccati dal modello inizialmente proposto dal programma e hanno sviluppato delle soluzioni originali adattate al contesto universitario svizzero. Queste esperienze hanno poi condotto ad una revisione del modello di introduzione dell'eLearning a livello del Campus Virtuale Svizzero: anziché puntare su corsi completamente on line si privilegiano i corsi ibridi, che mantengono una parte in presenza; inoltre il programma ha deciso di favorire la creazione di centri di supporto per l'eLearning a livello dell'intera università. Molte questioni restano tuttavia aperte in particolare per le modalità organizzative ed il modello economico dell'eLearning.

Parole chiave: apprendimento, computer, controllo della qualità, didattica, metodo di insegnamento, orientamento, tecnologie dell'informazione e della communicazione, valutazione, università 


\section{Institutional dynamics and project strategies in the Swiss Virtual Campus}

\section{Summary}

In this paper, we examine the development of Swiss Virtual Campus (SVC) projects and the interaction with their institutional context. We show that the views of the SVC program and of the universities on what eLearning is and how it should be implemented are different. In this context, SVC projects were led to partially abandon the original model proposed by the SVC towards the development of original solutions adapted to each use context. These experiences lead to a revision of the eLearning concept at the level of the whole program (towards a blended learning approach) and, to some extent, also to a change in implementation strategies, with the introduction of university support centers. However, we show that many questions are still open, especially on how to organize and to finance this eLearning model.

Key words: computer, didactics, evaluation, guidance, information and communications technology, learning, quality control, teaching method, university 
T h e m a 